\title{
Correction to: \\ Exosomal microRNAs Release as a Sensitive Marker for Drug-Induced Liver Injury In Vitro by Messner CJ, et al. Appl In Vitro Toxicol 2020:6;77-89. DOI: 10.1089/aivt.2020.0008
}

N THe SePtember 2020 issue of Applied In Vitro Toxicology (vol. 6, no. 3; 77-89), the article entitled "Exosomal microRNAs Release as a Sensitive Marker for Drug-Induced Liver Injury In Vitro” by Messner et al. requires correction.

An error was inadvertently introduced to the Abstract.

The original text read:

"Introduction: In vitro models for liver disease suffer from the lack of well-established and sensitive biomarkers of cellular damage. MicroRNAs (miRNAs; small noncoding RNAs) represent potential biomarkers for the detection of drug-induced liver injury in vitro and in vitro. Altered physiological state caused by disease or tissue damage results in altered release of exosomal-or protein-bound miRNAs, detectable in body fluids and cell culture media.",

The revised text now reads:

"Introduction: In vitro models for liver disease suffer from the lack of well-established and sensitive biomarkers of cellular damage. MicroRNAs (miRNAs; small noncoding RNAs) represent potential biomarkers for the detection of drug-induced liver injury in vivo and in vitro. Altered physiological state caused by disease or tissue damage results in altered release of exosomal-or protein-bound miRNAs, detectable in body fluids and cell culture media."

The online version of the article has been corrected to reflect this change.

The publisher apologizes for this error. 